\title{
NAC1, a potential stem cell pluripotency factor expression in normal endometrium, endometrial hyperplasia and endometrial carcinoma
}

\author{
MASAKO ISHIKAWA, KENTARO NAKAYAMA, SHAMIMA YEASMIN, \\ ATSUKO KATAGIRI, KOUJI IIDA, NAOMI NAKAYAMA and KOHJI MIYAZAKI \\ Departments of Obstetrics and Gynecology, Shimane University School of Medicine, \\ Enyacho 89-1, Izumo, Shimane 693-8501, Japan
}

Received August 11, 2009; Accepted October 8, 2009

DOI: 10.3892/ijo_00000591

\begin{abstract}
The purpose of this study was to investigate the role of NAC1 in the development of endometrial cancer. $\mathrm{NAC} 1$ expression and localization were assessed with immunohistochemistry in the normal cyclic human endometrium, hyperplastic endometrium, and endometrial cancer. Expression of NAC1 in the glandular cells was significantly higher in the early and mid proliferative phases than in the other menstrual phases, endometrial hyperplasia, and endometrial carcinoma. NAC1 expression was down-regulated during endometrial carcinogenesis. There were significant correlations between positive NAC1 expression and pathological grade $(\mathrm{P}=0.037)$. No significant associations were found between NAC1 expression and the other clinicopathological characteristics including patient age, FIGO staging, depth of myometrial invasion, pelvic lymph node metastasis, lymphovascular space invasion, menopause, or body mass index. NAC1 gene knockdown inhibited cell growth and induced apoptosis in Ishikawa, HHUA, and JHEM2 cell lines, all of which overexpressed NAC1. Ectopic overexpression of the NAC1 gene stimulated cell proliferation in the HEC1B, and JHEM1 endometrial cancer cell lines, which have lower endogenous NAC1 expression. Endometrial carcinomas with NAC1 overexpression are clinically aggressive, high-grade carcinomas. Therefore, detection of NAC1 overexpression in endometrial cancers may identify patients who will benefit from NAC1 targeted therapy.
\end{abstract}

\section{Introduction}

Endometrial cancer is the most common malignancy of the female genital tract and the fifth leading cancer in women

Correspondence to: Dr Kentaro Nakayama, Shimane University School of Medicine, Enyacho 89-1, Izumo, Shimane 693-8501, Japan E-mail:kn88@med.shimane-u.ac.jp

Abbreviations: BTB, bric-a-brac tramtrack broad complex

Key words: endometrial cancer, normal endometrium, NAC1, ovarian sex steroid worldwide (1). There are two main clinicopathologic variants of endometrial carcinoma (2). Type I tumors are usually estrogen-related. In general, they develop in pre- and perimenopausal women, and coexist with or are preceded by endometrial hyperplasia. In contrast, type II carcinomas are unrelated to estrogen, are associated with atrophic endometrium, and usually occur at an older age $(3,4)$. Four main molecular genetic alterations have been described in type I endometrial carcinomas, including microsatellite instability (MI), and mutations of the PTEN, K-ras, and B-catenin genes (2-5). These molecular alterations have also been described in atypical endometrial hyperplasia (2). Type II carcinomas, exemplified by serous papillary adenocarcinoma, have mutations of the p53 gene in approximately $90 \%$ of cases $(2,6)$. The full spectrum of genetic alterations in endometrial cancer, however, has not been defined.

Normal endometrial glandular cells and a subset of cases of endometrial hyperplasia and carcinomas express estrogen receptors (ER) and progesterone receptors (PR). Endometrial cells are subject to hormone-induced growth regulation. Aspects of this include the up-regulation and down-regulation of steroid receptors $(7,8)$, and the involvement of growth factors or cytokines (9). Although sex steroid-induced growth regulation is generally thought to be enacted through ER and $\mathrm{PR}$, the molecular pathways downstream of the receptors that eventually promote the transcription of target genes have not been fully elucidated. Steroid receptor cofactors have recently been identified as important molecules intervening between the receptors and target genes. These cofactors are now functionally divided into two subclasses, coactivators, and corepressors. Cofactors are either activators or repressors for other transcription factors and participate in a transcriptional factor cascade of positive and negative regulators. Only a few factors, however, are directly involved in endometrial cancer.

The genes of the BTB/POZ family participate in several cellular functions including proliferation, apoptosis, transcription control, and cell morphology maintenance (10). The BTB/POZ proteins share an evolutionally conserved BTB/ $\mathrm{POZ}$ protein-protein interaction motif at the $\mathrm{N}$-terminal that mediates either homodimer or heterodimer formation (10-12). Some BTB/POZ proteins mediate transcriptional repression through their ability to recruit corepressors (13-16). 
By serial analysis of gene expression (SAGE) of all 130 deduced human $\mathrm{BTB} / \mathrm{POZ}$ genes, we identified $\mathrm{NAC} 1$ as a carcinoma-associated $\mathrm{BTB} / \mathrm{POZ}$ gene (17). NAC1 is a transcription repressor that is involved in self renewal and the maintenance of pluripotency in embryonic stem cells (18). $\mathrm{NAC1}$ is significantly overexpressed in several types of human carcinomas (17), where it plays a critical role in maintaining tumor cell proliferation and survival $(17,19-21)$. Recently, we showed that NAC1 is essential for the proliferation of normal cyclic endometrial glandular cells and is up-regulated by estrogen (22). However, NAC1 expression has not yet been investigated in the context of endometrial cancer. The purpose of this study was to investigate the role of NAC1 in the development of endometrial cancer.

\section{Materials and methods}

Tissue samples and immunohistochemistry. A total of 126 paraffin-embedded tumor tissue and normal endometrial tissue samples were obtained from the Department of Obstetrics and Gynecology at Shimane University Hospital. Samples consisted of 66 endometrial carcinomas, six endometrial hyperplasia samples and 54 normal endometrial tissue samples. Cancer patients had received appropriate therapy at Shimane University Hospital between January 1998 and December 2008. Acquisition of tissue specimens and clinical information was approved by the institutional review board of Shimane University. Endometrial carcinomas were classified according to the surgical staging system of the International Federation of Gynecology and Obstetrics (FIGO). Invasive carcinomas were divided into 32 cases of stage I disease, 24 of stage II disease, 20 of stage III disease, and eight of stage IV disease. All tumors were classified histologically according to World Health Organization criteria. The median patient age was 60 years (range 26-84 years). The primary surgery for the 66 patients with invasive carcinomas was as follows: total hysterectomy in 16 patients; modified radical hysterectomy in 46 ; radical hysterectomy in four. Systemic retroperitoneal lymphadenectomy was carried out in approximately $90 \%$ of the patients. Patients with risk factors for recurrence (such as deep myometrial invasion, cervical invasion, lymph node metastasis, lymphovascular space invasion, or positive peritoneal cytology) underwent external radiotherapy and/or 4-6 cycles of CAP therapy (cisplatin $100 \mathrm{mg} / \mathrm{m}^{2}$, doxorubicine $40 \mathrm{mg} / \mathrm{m}^{2}$, cyclophosphamide $500 \mathrm{mg} / \mathrm{m}^{2}$ ) or TC therapy (paclitaxel $175 \mathrm{mg} / \mathrm{m}^{2}$; carboplatin, according to Chatelut's formula (area under curve $=5 \mathrm{mg} / \mathrm{m}^{2}$ ) as a postoperative adjuvant therapy. All 66 patients with invasive endometrial carcinoma were followed for survival analysis. The follow-up period ranged from 5 to 90 months, with a median of 39 months.

Fifty-four paraffin-embedded endometrial tissues were obtained from the Department of Obstetrics and Gynecology at Shimane University Hospital. All normal endometrial tissues were taken from patients who had undergone hysterectomy for non-endometrial gynecological disorders (i.e., uterine leiomyoma, early cervical cancer, and ovarian cancer). None of the study participants had taken exogenous hormones prior to surgery. All patients had regular menstrual cycles. Diagnosis was based on conventional morphological examination of $\mathrm{H} \& \mathrm{E}$ stained sections. All samples were classified according to the criteria described by Noyes et al (23). Endometrial samples meeting the inclusion criteria were classified into five groups: menstrual $(n=4)$, early proliferative $(n=10)$, mid proliferative $(n=5)$, late proliferative $(n=10)$, early-mid secretory $(n=11)$, and late secretory $(n=14)$. The paraffin tissues were organized into tissue microarrays, which were made by removing $3 \mathrm{~mm}$ diameter cores of tumor from each block.

Immunohistochemistry was performed on deparaffinized sections using the NAC1 antibody at a dilution of 1:100 (Novus Biologicals, Littleton, CO) and an EnVision+System peroxidase kit (Dako, Carpinteria, CA). After antigen retrieval in a sodium citrate buffer, slides were incubated overnight at $4^{\circ} \mathrm{C}$ with each antibody. The slides for all samples were evaluated with a light microscope by two researchers in a blinded manner relating to the menstrual cycle phase. The antibody staining intensity was then analyzed in the glands and stroma using the HSCORE (24). This modified HSCORE is calculated as follows: HSCORE $=\Sigma \mathrm{P}_{i}(i)$, where $i$ is the intensity of staining $(0=$ undetectable, $1=$ weakly positive, $2=$ moderately positive, $3=$ intensely positive) and $\mathrm{P}_{I}$ is a score that is based on the percentage of stained cells for each intensity, varying from $0-100 \%$.

Cell culture and treatment. Ishikawa 3-H-12 cells were provided by Dr Masato Nishida (Kasumigaura Medical Center, Ibaragi Japan). HHUA, JHEM1, and JHEM2 cells were also obtained from Riken Bioresource Center (Ibaragi Japan). HEC1B cells were also obtained from Health Science Research Resources Bank (Tokyo Japan). All of the cells were maintained in DMEM (Life Technologies, Gaithersburg, MD) supplemented with $10 \%$ fetal bovine serum, 100 units $/ \mathrm{ml}$ of penicillin, and $100 \mu \mathrm{g} / \mathrm{ml}$ of streptomycin.

Western blot analysis. Cell lysates were prepared by dissolving cell pellets in Laemmli sample buffer (BioRad, Hercules, CA) supplemented with 5\% ß-mercaptoethanol (Sigma). Western blot analysis was performed on normal endometrial cultures. Similar amounts of total protein from each lysate were loaded and separated on $10 \%$ Tris-Glycine-SDS polyacrylamide gels (Novex, San Diego, CA) and electroblotted to Millipore Immobilon-P polyvinylidene difluoride membranes. The membranes were probed with the NAC1 antibody $(1: 100)$ followed by a peroxidase conjugated anti-mouse immunoglobulin $(1: 10,000)$. The same membrane was probed with an antibody that reacted with GAPDH for loading controls. Western blots were developed by chemiluminescence (Pierce, Rockford, IL).

siRNA knockdown of NACl gene expression. Two small interfering RNA sequences (siRNAs) that targeted NAC1 were designed, with sense sequences of UGAUGUACACG UGGUGCCUGUCACCA and GAGGAAGAACUCGGUG CCCUUCUCCAU. Control siRNA (luciferase siRNA) was purchased from IDT (Coralville, IA). Cells were seeded into 96-well plates and transfected with siRNAs using oligofectamine (Invitrogen). Cell number was determined indirectly by an MTT assay $72 \mathrm{~h}$ after transfection of siRNA (25). Data were expressed as the mean \pm SD from triplicates. Apoptotic cells were detected using Annexin V staining. Data were expressed as the mean $\pm \mathrm{SD}$ from triplicates. 

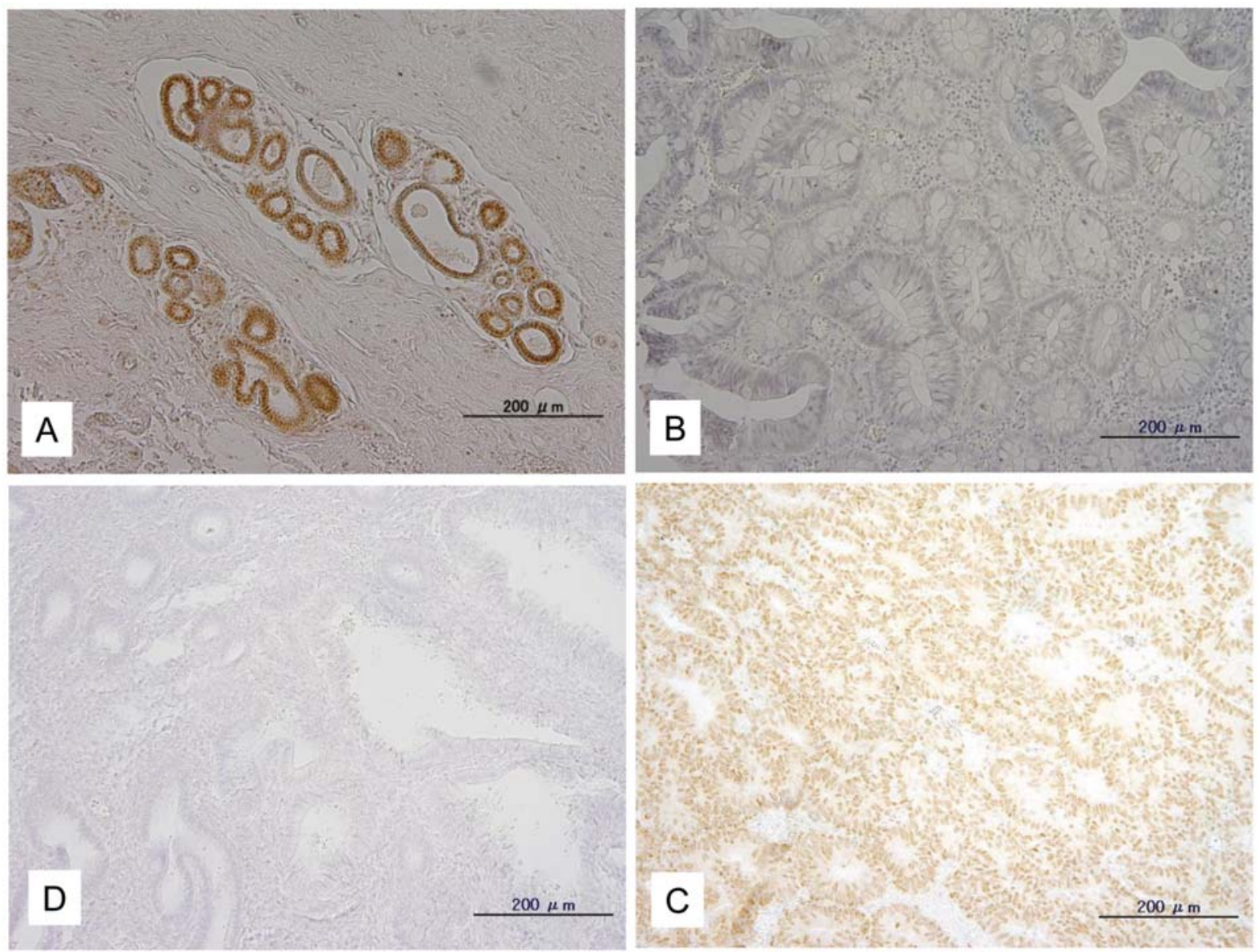

Figure 1. Immunoreactivity of NAC1 in normal endometrial tissues, hyperplasia and carcinoma. Intense immunoreactivity is present in the nuclei of normal endometrial glandular cells. (A) A case with strong staining of NAC1 in the early proliferative phase (upper left). (B and C) NAC1 immunointensity is negative in both hyperplasia (upper right) and grade 1 endometrial carcinoma (lower left). (D) A case with strong staining of NAC1 in grade 3 endometrial carcinoma (lower right).

Retrovirus transfection and generation of NAC1. The NAC1 retrovirus vector ( $\mathrm{pWZL}-$ Hygro retroviral vector) was kindly provided by Dr Ie-Ming Shih (Johns Hopkins Medical Institutions, Baltimore, MD). Packaging cells (Phoenix cells) were transiently transfected with the NAC1 or empty vector using the lipofectamine method (Invitrogen). On the following day, the supernatant was harvested and passed through a $0.45 \mu \mathrm{mol} / 1$ syringe filter. The filtered viral supernatant was resupended in $4 \mu \mathrm{g} / \mathrm{ml}$ polybrene and added to cancer cell culture. Twenty-four hours after infection, cells were harvested and used for assays.

Statistical analysis. Results are expressed as the mean \pm SEM. Student's t-test (for comparison of two groups) or one-way analysis of variance (ANOVA; for comparison of more than two groups) was used to evaluate numeric data. The $\chi^{2}$ test or Fischer's exact test was used for comparisons of categorical data. The correlation coefficient ( $r$ ) between different parameters was determined by simple regression analysis. A $\mathrm{P}<0.05$ was considered statistically significant. Patient survival was calculated from the date of diagnosis to the date of death or last follow up. The data were plotted as Kaplan-Meier curves, and the statistical significance was determined by the log-rank test. Data were censored when patients were lost to follow-up.

\section{Results}

Expression of NAC1 in normal endometria, hyperplasia, and endometrial carcinoma. Immunohistochemistry for NAC1 of the normal endometrium sections revealed strong staining of the glands in both the proliferative phases (Fig. 1). NAC1 protein was detected in the gland cell nuclei. Expression of NAC1 in the gland cells was significantly higher in the early and mid proliferative phases than in the other menstrual phases, hyperplasia, and carcinoma (Fig. 2). Expression levels of NAC1 in endometrial carcinoma and hyperplasia were significantly lower than that in normal cyclic endometrium.

Relationship between clinicopathological findings and NAC1 expression in endometrial cancer. Table I summarizes the relationship between clinicopathological findings and the expression of NAC1 in endometrial carcinomas. There was a significant correlation between positive NAC1 expression and pathological grade $(\mathrm{P}=0.037)$. No significant associations were found between NAC1 expression and the other clinicopathological characteristics including patient age, FIGO staging, depth of myometrial invasion, pelvic lymph node metastasis, lymphovascular space invasion, menopause, body mass index, or status of the residual tumor (Table I). Next, 
Table I. Association between NAC1 expression and clinicopathological factors in patients with endometrial cancer.

\begin{tabular}{|c|c|c|c|c|}
\hline \multirow[t]{2}{*}{ Factors } & \multirow[t]{2}{*}{ Patients } & \multicolumn{2}{|c|}{ NAC1 expression } & \multirow[t]{2}{*}{ P-value } \\
\hline & & Negative & Positive & \\
\hline \multicolumn{5}{|l|}{ Figo stage } \\
\hline $\mathrm{I}$ & 48 & 27 & 21 & 0.6497 \\
\hline II, III, IV & 18 & 9 & 9 & \\
\hline \multicolumn{5}{|l|}{ Grade } \\
\hline G1 & 29 & 20 & 9 & 0.0373 \\
\hline G2, G3 & 37 & 16 & 21 & \\
\hline \multicolumn{5}{|c|}{ Lymph node metastasis } \\
\hline Negative & 63 & 35 & 28 & 0.4501 \\
\hline Positive & 3 & 1 & 2 & \\
\hline \multicolumn{5}{|c|}{ Depth (myometrial invasion) } \\
\hline $\mathrm{a}, \mathrm{b}$ & 46 & 24 & 22 & 0.5573 \\
\hline $\mathrm{c}$ & 20 & 12 & 8 & \\
\hline \multicolumn{5}{|c|}{ Lymphovascular space invasion } \\
\hline Negative & 45 & 26 & 19 & 0.4401 \\
\hline Positive & 21 & 10 & 11 & \\
\hline \multicolumn{5}{|l|}{ Menopause } \\
\hline Peri, pre & 46 & 25 & 21 & 0.961 \\
\hline Post & 20 & 11 & 9 & \\
\hline \multicolumn{5}{|c|}{ Body mass index } \\
\hline$<25$ & 54 & 28 & 26 & 0.3512 \\
\hline$\geq 25$ & 12 & 8 & 4 & \\
\hline \multicolumn{5}{|l|}{ Age (years) } \\
\hline$<60$ & 40 & 34 & 6 & 0.2467 \\
\hline$\geq 60$ & 48 & 36 & 12 & \\
\hline
\end{tabular}

we examined the prognostic effect of NAC1 expression in endometrial cancer. There was no significant relationship between NAC1 expression and overall/disease-free survival in patients with endometrial carcinoma (data not shown).

Functional analysis of NACl expression in endometrial cancer cells. Since some high grade endometrial cancers do overexpress NAC1, we tested whether NAC1 could represent a therapeutic target. NAC1 siRNA was applied to the culture medium of Ishikawa, HHUA, JHEM2, HEC1B and JHEM1 endometrial cancer cells. siRNA treatment significantly reduced NAC1 protein expression compared with control siRNA treatment (Fig. 3A). NAC1 siRNA reduced cell numbers significantly in Ishikawa, HHUA, and JHEM2 cells that overexpressed NAC1 (Fig. 3A). This reduction in cell number was not seen in the HEC1B and JHEM1 cells that expressed lower levels of NAC1 $(\mathrm{P}<0.05$, Student's t-test $)$ (Fig. 3B). The inhibition of cell growth following repression of NAC1 expression in Ishikawa, HHUA, and JHEM2 likely resulted from the induction of apoptosis. The percentage of apoptotic cells identified using Annexin V staining was significantly increased in the NAC1 siRNA-treated cells compared with control siRNA-treated cells (Fig. 4). Next, to confirm

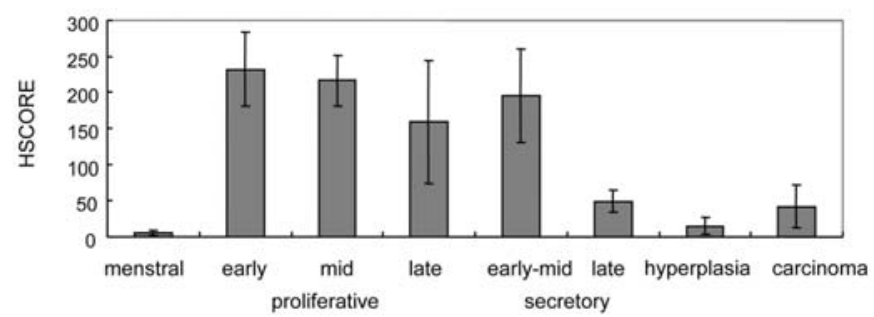

Figure 2. Distribution pattern of NAC1 expression across the menstrual cycle, hyperplasia and carcinoma.

the results of NAC1 knockdown experiments, we generated NAC1 stable expression cells from HEC1B and JHEM1 cells which normally had lower endogenous NAC1 expression. These cells were stably transfected with a NAC1 expression retroviral vector. When compared with vector-transfected controls, HEC1B and JHEM1 cells that expressed NAC1 had higher cellular proliferation based on growth assay results (Fig. 5). 
B

A

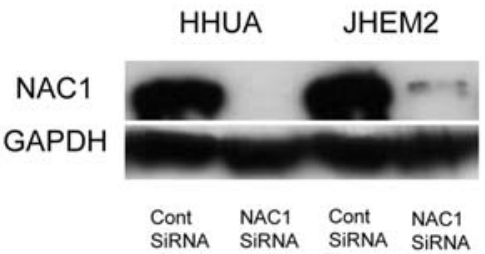

C

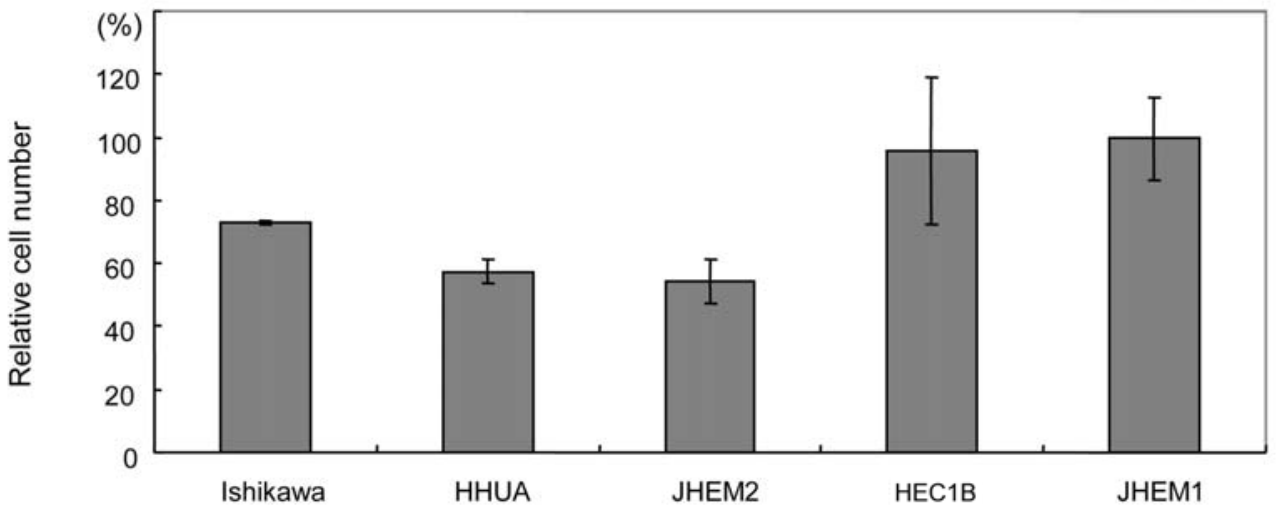

Figure 3. Effects of NAC1 knockdown on cell proliferation and apoptosis in endometrial cancer cell lines. (A) Western blot analysis showing a higher expression level of NAC1 protein in Ishikawa, HHUA and JHEM2 cells than in HEC1B and JHEM1 cells. (B) Western blot analysis showing a significant reduction of NAC1 protein in NAC1 siRNA-treated cells compared with control siRNA-treated cells in HHUA and JHEM2 cells. NAC1 siRNA significantly reduces the cell number in Ishikawa cells. (C) NAC1 siRNA significantly reduces cell numbers in cell lines with NAC1 overexpression including Ishikawa, HHUA and JHEM2 compared with cell lines without NAC1 overexpression.

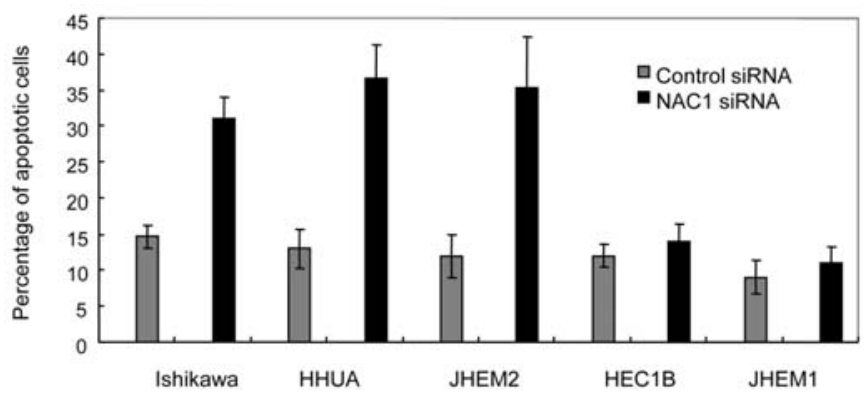

Figure 4. Detection of apoptotic cells. (A) The experiment was performed $72 \mathrm{~h}$ after NAC1 siRNA or control siRNA treatment. Treatment with NAC1 siRNA increases apoptosis of Ishikawa, HHUA, and JHEM2 cells as measured by Annexin V staining.

\section{Discussion}

Previously, we reported that NAC1 was overexpressed in the normal endometrium in the early and mid proliferative phases. Expression was up-regulated by E2 and was associated with E2-induced cell growth in endometrial cells (22). Prolonged exposure to estrogen is one of the risk factors for the development of endometrial cancer, a malignancy that is becoming increasingly common (26). Although the effects of estrogen on the proliferation of endometrial cells are complex, upregulation of $\mathrm{NAC} 1$ by estrogen may contribute to estrogen induced endometrial carcinogenesis. Therefore, in this study, we tried to clarify the relationship between estrogen-induced endometrial carcinogenesis and NAC1 expression using endometrial cancer specimens. Ours is the first report describing the cellular distribution of NAC1 expression in human normal, hyperplastic, and malignant endometrial tissue. Unexpectedly, the results indicate that a stepwise reduction in NAC1 protein expression is significantly associated with the progression from normal endometrium to hyperplasia and finally to carcinoma. The relationship between NAC1 expression and cancer initiation or progression is unclear. Atypical endometrial hyperplasia is thought to be a precursor of endometrial cancer, with the potential to progress to invasive endometrial cancer in approximately $29 \%$ of cases (27). Surveillance of the hyperplastic endometrium is presently limited by the inability to predict the risk of progression. The stepwise downregulation of NAC1 expression may reflect a loss of estrogen signaling in endometrial hyperplasia and cancer and may explain the reason hormone therapy is not effective for endometrial cancer (28).

In endometrial cancer, the most significant factors determining a patient's prognosis are histological grade, clinical stage, and myometrial invasion (29). In the current study, endometrial carcinomas with NAC1 overexpression were all 
A

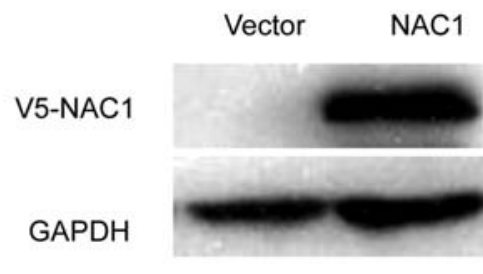

HEC1B

C

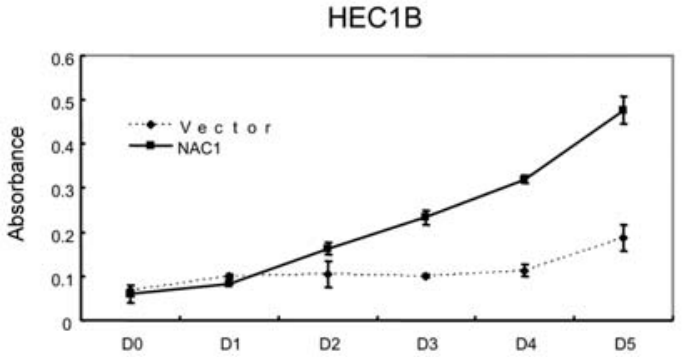

B

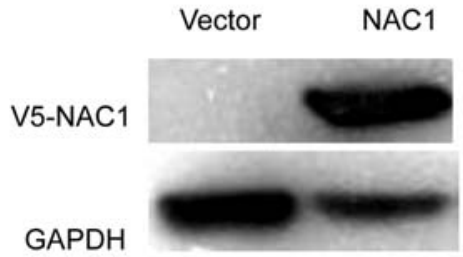

JHEM1

$\mathrm{D}$

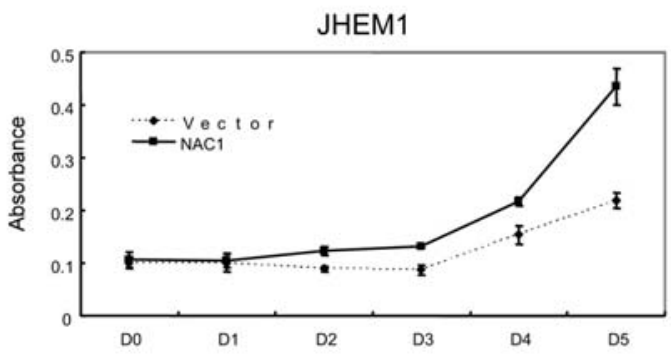

Figure 5. Functional analysis of NAC1 expression. (A and B) Western blot analysis shows that NAC1-transfected HEC1B and JHEM1 express NAC1 protein. Anti-V5 antibody was used to detect NAC1 protein. (C and D) The NAC1 stable transfected HEC1B and JHEM1 cells demonstrate higher proliferative activity than the vector-only controls as evidenced by a time-dependent increase in cell number in low $(0.5 \%)$ serum-containing medium.

clinically aggressive, high-grade carcinomas $(30,31)$. Thus, NAC1 may represent a therapeutic target. The phenotypic changes were seen only in endometrial cancer cells with NAC1 overexpression, not in those with low NAC1 expression. Reduction of NAC1 expression resulted in apoptosis in the NAC1 expressing cell lines Ishikawa, HHUA, and JHEM2, indicating that NAC1 was essential for proliferation and survival of these cell lines. These results are similar to those obtained in our previous experiments in ovarian and cervical cancer cell lines $(17,20)$. Besides its critical role in maintaining cell proliferation and survival, NAC1 overexpression could also enhance cellular proliferation. These findings suggest that NAC1 is a gene with significant cell growth and survival effects in endometrial carcinomas, particularly high grade tumors.

Taken together, the current and previous findings suggest that NAC1 may have different functions in normal cyclic endometrium and endometrial cancer. NAC1 may play an important role in estrogen-induced cell growth in normal cyclic endometrium. It may be less important in endometrial cancers that have lost estrogen signaling and are stimulated by other oncogenic pathways $(28,32,33)$. While many endometrial cancers lose NAC1 expression, some high grade tumors continue to produce it. This may be explained by the known positive effects of NAC1 on tumor growth and survival $(17,20)$. During endometrial carcinogenesis, tumors lose estrogen signaling with concomitant loss of NAC1 expression. However, as tumors become more aggressive, they may reexpress NAC1 thereby gaining a survival advantage.

Recently, new evidence has shown a role of NAC1 as an important transcriptional regulator that functions as part of an extended regulatory network necessary for preserving the pluripotent state of ES cells $(18,34)$. NAC1 is a primary Nanog interacting protein that is part of the protein regulatory complex responsible for maintaining pluripotency (18). The NAC1 protein complex has been shown to transcriptionally regulate approximately 800 genes in ES cells (34). Interestingly, NAC1 itself has been shown to regulate transcription of the transcription factors, Nanog, Oct4 and Sox2, which are essential for the development and maintenance of the pluripotent state of ES cells (34). Given that NAC1 likely participates in transcriptional regulation of these genes in the maintenance of cell pluripotency, it is plausible that NAC1 may contribute to cell growth and survival by enhancing stem-cell like features of high grade endometrial cancer cells.

In summary, the present study demonstrated that NAC1 was overexpressed in the normal cyclic endometrium in the early and mid proliferative phases. The fact that NAC1 expression was down-regulated in endometrial cancer may be related to loss of estrogen signaling in development of endometrial cancer. NAC1 expression was essential for growth and survival in some high grade endometrial cancers. NAC1 therefore is a potential therapeutic target. Endometrial carcinomas with NAC1 overexpression are clinically aggressive, high-grade carcinomas $(30,31)$, therefore, detection of NAC1 overexpression in endometrial cancers may identify patients who will benefit from NAC1 targeted therapy.

\section{Acknowledgements}

This study was supported by grants from the Ministry of Education, Culture, Sports, Science and Technology in Japan and the Japan Society of Gynecologic Oncology. 


\section{References}

1. Pisani P, Bray F and Parkin DM: Estimates of the world-wide prevalence of cancer for 25 sites in the adult population. Int J Cancer 97: 72-81, 2002.

2. Shiozawa T and Konishi I: Early endometrial carcinoma: clinicopathology, hormonal aspects, molecular genetics, diagnosis, and treatment. Int J Clin Oncol 11: 13-21, 2006.

3. Matias-Guiu X, Catasus L, Bussaglia E, Lagarda H, Garcia A, Pons C, Munoz J, Arguelles R, Machin P and Prat J: Molecular pathology of endometrial hyperplasia and carcinoma. Hum Pathol 32: 569-577, 2001

4. Lax SF: Molecular genetic pathways in various types of endometrial carcinoma: from a phenotypical to a molecular-based classification. Virchows Arch 444: 213-223, 2004.

5. Tashiro H, Blazes MS, Wu R, Cho KR, Bose S, Wang SI, Li J, Parsons R and Ellenson LH: Mutations in PTEN are frequent in endometrial carcinoma but rare in other common gynecological malignancies. Cancer Res 57: 3935-3940, 1997.

6. Tashiro H, Isacson C, Levine R, Kurman RJ, Cho KR and Hedrick L: p53 gene mutations are common in uterine serous carcinoma and occur early in their pathogenesis. Am J Pathol 150: $177-185,1997$

7. Levy C, Robel P, Gautray JP, De Brux J, Verma U, Descomps B and Baulieu EE: Estradiol and progesterone receptors in human endometrium: normal and abnormal menstrual cycles and early pregnancy. Am J Obstet Gynecol 136: 646-651, 1980.

8. Lessey BA, Killam AP, Metzger DA, Haney AF, Greene GL and McCarty KS Jr: Immunohistochemical analysis of human uterine estrogen and progesterone receptors throughout the menstrual cycle. J Clin Endocrinol Metab 67: 334-340, 1988.

9. Giudice LC: Growth factors and growth modulators in human uterine endometrium: their potential relevance to reproductive medicine. Fertil Steril 61: 1-17, 1994.

10. Stogios PJ, Downs GS, Jauhal JJ, Nandra SK and Prive GG: Sequence and structural analysis of BTB domain proteins. Genome Biol 6: R82, 2005.

11. Bardwell VJ and Treisman R: The POZ domain: a conserved protein-protein interaction motif. Genes Dev 8: 1664-1677, 1994.

12. Albagli O, Dhordain P, Deweindt C, Lecocq G and Leprince D: The BTB/POZ domain: a new protein-protein interaction motif common to DNA- and actin-binding proteins. Cell Growth Differ 6: 1193-1198, 1995

13. David G, Alland L, Hong SH, Wong CW, De Pinho RA and Dejean A: Histone deacetylase associated with $\mathrm{mSin} 3 \mathrm{~A}$ mediates repression by the acute promyelocytic leukemia-associated PLZF protein. Oncogene 16: 2549-2556, 1998.

14. Dhordain P, Albagli O, Lin RJ, Ansieau S, Quief S, Leutz A, Kerckaert JP, Evans RM and Leprince D: Corepressor SMRT binds the BTB/POZ repressing domain of the LAZ3/BCL6 oncoprotein. Proc Natl Acad Sci USA 94: 10762-10767, 1997.

15. Huynh KD and Bardwell VJ: The BCL-6 POZ domain and other POZ domains interact with the co-repressors N-CoR and SMRT. Oncogene 17: 2473-2484, 1998.

16. Melnick A, Carlile G, Ahmad KF, Kiang CL, Corcoran C, Bardwell V, Prive GG and Licht JD: Critical residues within the BTB domain of PLZF and Bcl-6 modulate interaction with corepressors. Mol Cell Biol 22: 1804-1818, 2002.

17. Nakayama K, Nakayama N, Davidson B, Sheu JJ, Jinawath N, Santillan A, Salani R, Bristow RE, Morin PJ, Kurman RJ, Wang TL and Shih IeM: A BTB/POZ protein, NAC-1, is related to tumor recurrence and is essential for tumor growth and survival. Proc Natl Acad Sci USA 103: 18739-18744, 2006.
18. Wang J, Rao S, Chu J, Shen X, Levasseur DN, Theunissen TW and Orkin SH: A protein interaction network for pluripotency of embryonic stem cells. Nature 444: 364-368, 2006.

19. Davidson B, Berner A, Trope CG, Wang TL and Shih IeM: Expression and clinical role of the bric-a-brac tramtrack broad complex/poxvirus and zinc protein NAC-1 in ovarian carcinoma effusions. Hum Pathol 38: 1030-1036, 2007.

20. Yeasmin S, Nakayama K, Ishibashi M, Katagiri A, Iida K, Purwana IN, Nakayama N and Miyazaki K: Expression of the Bric-a-Brac tramtrack broad complex protein NAC-1 in cervical carcinomas seems to correlate with poorer prognosis. Clin Cancer Res 14: 1686-1691, 2008.

21. Ishibashi M, Nakayama K, Yeasmin S, Katagiri A, Iida K, Nakayama N, Fukumoto M and Miyazaki K: A BTB/POZ gene, NAC-1, a tumor recurrence-associated gene, as a potential target for Taxol resistance in ovarian cancer. Clin Cancer Res 14: 3149-3155, 2008.

22. Ishibashi M, Nakayama K, Yeasmin S, Katagiri A, Iida K Nakayama N and Miyazaki K: Expression of a BTB/POZ protein, $\mathrm{NAC} 1$, is essential for the proliferation of normal cyclic endometrial glandular cells and is up-regulated by estrogen. Clin Cancer Res 15: 804-811, 2009.

23. Noyes RW, Hertig AT and Rock J: Dating the endometrial biopsy. Am J Obstet Gynecol 122: 262-263, 1975.

24. Mao TL, Seidman JD, Kurman RJ and Shih IeM: Cyclin E and p16 immunoreactivity in epithelioid trophoblastic tumor - an aid in differential diagnosis. Am J Surg Pathol 30: 1105-1110, 2006.

25. Nakayama K, Miyazaki K, Kanzaki A, Fukumoto M and Takebayashi Y: Expression and cisplatin sensitivity of coppertransporting P-type adenosine triphosphatase (ATP7B) in human solid carcinoma cell lines. Oncol Rep 8: 1285-1287, 2001.

26. Sutton G: Hormonal aspects of endometrial cancer. Curr Opin Obstet Gynecol 2: 69-73, 1990.

27. Kurman RJ, Kaminski PF and Norris HJ: The behavior of endometrial hyperplasia. A long-term study of 'untreated' hyperplasia in 170 patients. Cancer 56: 403-412, 1985.

28. Uchikawa J, Shiozawa T, Shih HC, Miyamoto T, Feng YZ, Kashima H, Oka K and Konishi I: Expression of steroid receptor coactivators and corepressors in human endometrial hyperplasia and carcinoma with relevance to steroid receptors and $\mathrm{Ki}-67$ expression. Cancer 98: 2207-2213, 2003.

29. Takai N, Miyazaki T, Nishida M, Shang S, Nasu K and Miyakawa I: Clinical relevance of Elf-1 overexpression in endometrial carcinoma. Gynecol Oncol 89: 408-413, 2003.

30. Sorosky JI: Endometrial cancer. Obstet Gynecol 111: 436-447, 2008.

31. Uharcek P: Prognostic factors in endometrial carcinoma. J Obstet Gynaecol Res 34: 776-783, 2008.

32. Sasaki M, Kotcherguina L, Dharia A, Fujimoto S and Dahiya R: Cytosine-phosphoguanine methylation of estrogen receptors in endometrial cancer. Cancer Res 61: 3262-3266, 2001.

33. Woo MM, Alkushi A, Verhage HG, Magliocco AM, Leung PC, Gilks CB and Auersperg N: Gain of OGP, an estrogen-regulated oviduct-specific glycoprotein, is associated with the development of endometrial hyperplasia and endometrial cancer. Clin Cancer Res 10: 7958-7964, 2004.

34. Kim J, Chu J, Shen X, Wang J and Orkin SH: An extended transcriptional network for pluripotency of embryonic stem cells. Cell 132: 1049-1061, 2008. 